\title{
Polycrystalline Time-of-Flight Inelastic Neutron Scattering beyond the Density of States ${ }^{+}$
}

\author{
Michael Leitner \\ Heinz Maier-Leibnitz Zentrum (MLZ), Garching/Technische Universität München, \\ 85748 Garching, Germany; michael.leitner@frm2.tum.de \\ + Presented at the 37th International Symposium on Dynamical Properties of Solids (DyProSo 2019), Ferrara, \\ Italy, 8-12 September 2019.
}

Published: 5 September 2019

Conventionally, experimental phonon dispersions are determined by inelastic neutron scattering on triple-axis spectrometers or by inelastic $X$-ray scattering, in both cases requiring single crystalline samples. When only polycrystals are available, the energy-dependent density of states (DOS) can be measured as an alternative.

Here I will make the point that the $(|\mathbf{Q}|, E)$-dependent spectral density, which is the primary quantity measured in coherent time-of-flight scattering on polycrystals, has a much greater information content than the DOS, to which it is customarily reduced, and that this information can be accessed by modelling the scattering signal. I will present applications of this technique to different systems and specifically show how the efficiency of the method makes it possible to perform temperature-dependent measurements with fine resolution, such as the behaviour of the phonon frequencies around the $\alpha-\gamma$ transition in elemental iron.
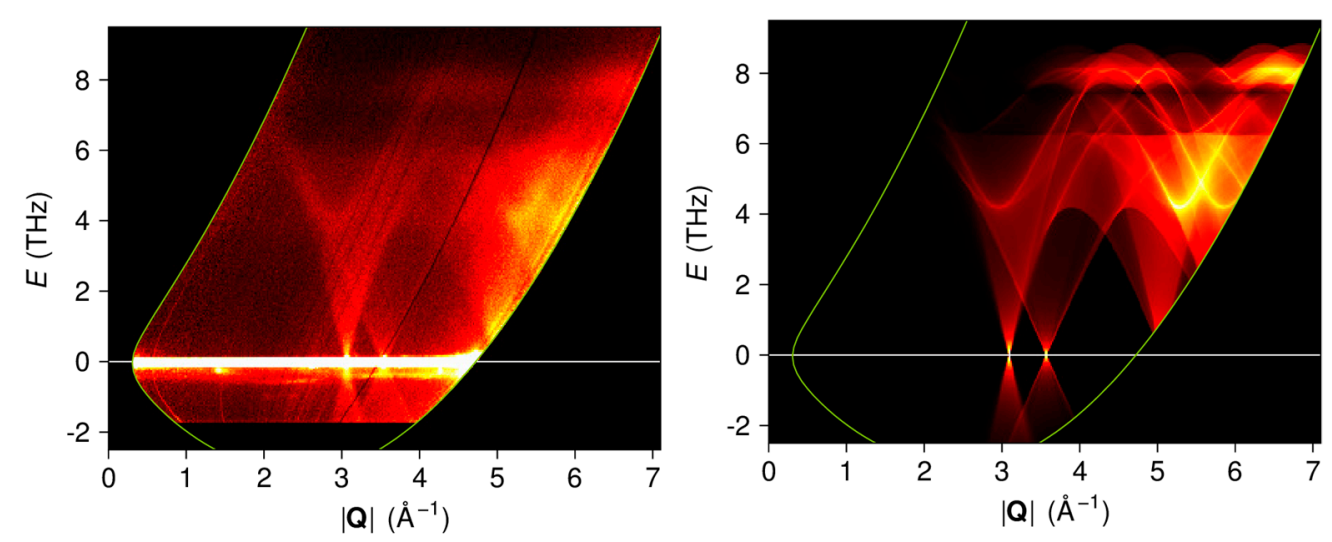

Figure 1. Time-of-flight spectra of polycrystalline Nickel: measured (left) and simulated (right).

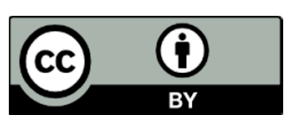

(C) 2019 by the author. Licensee MDPI, Basel, Switzerland. This article is an open access article distributed under the terms and conditions of the Creative Commons Attribution (CC BY) license (http://creativecommons.org/licenses/by/4.0/). 